\title{
CONSIDERACIONES ACERCA DE LA ENSEÑANZA DE LOS RASGOS FONO-PROSÓDICOS DEL FRANCÉS COMO LENGUA EXTRANJERA
}

\author{
Luis Eyén Reina García ${ }^{1}$ \\ Frédéric Torterat ${ }^{2}$ \\ Ramón Luis Herrera Rojas ${ }^{3}$
}

Recibido 21/05/2019 Aceptado 12/06/2019

1. Facultad de Ciencias Pedagógicas. Universidad de Sancti Spíritus "José Martí Pérez",

Máster en Ciencias de la Educación, Profesor Auxiliar. Ireina@uniss.edu.cu

2. Profesor Catedrático de la Universidad de Montpellier, Facultad de Educación, Doctor en Ciencias del Lenguaje. frederic.torterat@umontpellier.fr

3. Facultad de Humanidades Universidad de Sancti Spíritus "José Martí Pérez", Doctor en Ciencias Filológicas, Profesor Titular.rluis@uniss.edu.cu
Este trabajo constituye un breve análisis acerca dela enseñanza de los rasgos fono-prosódicos del francés como lengua extranjera en la carrera Licenciatura en Educación, Lenguas Extranjeras, en la Universidad de Sancti Spíritus "José Martí Pérez", de Cuba. Se parte de las concepciones del contexto cubano para la enseñanza de las lenguas extranjeras, los aportes teóricos que ofrecen los clásicos de la didáctica de la pronunciación del francés. Se asume que la pronunciación es una habilidad que ha de enseñarse y desarrollarse en el proceso de enseñanza-aprendizaje de la LE para que el estudiante, como parte de su competencia comunicativa, alcance una competencia fónica. Finalmente se proponen tres actividades que permiten demostrar lo visto en la teoría investigada.

4 This work constitutes a brief analysis of the teaching of the phono-prosodic traits of French as a foreign language in the Bachelor's degree in Education, Foreign Languages, at the University of Sancti Spíritus "José Martí Pérez", Cuba. It is based on the conceptions of the Cuban context for the teaching of foreign languages, the theoretical contributions offered by the classics of the didactics of French pronunciation. It is assumed that pronunciation is a skill that has to be taught and developed in the teaching-learning process of LE so that the student, as part of his communicative competence, reaches a phonic competence. Finally, three activities are proposed to demonstrate what has been seen in the investigated theory.

DOI

https://doi.org/10.15366/didacticas2019.20.007

PALABRAS CLAVE

Enseñanza; Lenguas extranjeras; Pronunciación; Rasgos fono-prosódicos

KEYWORDS

Teaching; Foreign languages; Pronunciation; Phono-prosodic traits 


\section{INTRODUCCIÓN}

La necesidad de enseñar las lenguas extranjeras a las nuevas generaciones acorde a las exigencias sociales, enmarcadas en las circunstancias históricas, pone en el centro de las investigaciones científicas a la didáctica de la pronunciación.

La comunicación es esencialmente oral. Sobre el mismo tema, pero referido a la enseñanza de la lengua extranjera (LE), muchos autores reconocen la primacía del lenguaje oral que se ha basado en la realidad objetiva de su naturaleza como fenómeno social y medio de comunicación verbal por excelencia. Por tanto, la prioridad se le da al establecimiento de mecanismos de comprensión y producción oral. La escritura, salvo en cursos de especialización en el idioma, tiene un carácter instrumental o auxiliar, más que un fin en sí misma.

En el caso de la enseñanza de la pronunciación del francés, es importante partir de los presupuestos teóricos y metodológicos que existen acerca de la fonética correctiva, articulatoria y todo el tratamiento a los rasgos segmentales (realizaciones fónicas) y suprasegmentales (ritmo, entonación, acentuación) de la lengua francesa, para un público hispanohablante. Con este fin se propone el análisis de algunos elementos acerca de la enseñanza de los rasgos fono-prosódicos del francés como lengua extranjera en el contexto cubano. Es además, el resultado de una tesis de doctorado en la Universidad de Sancti Spíritus "José Martí Pérez", en Cuba que se lleva a cabo por el autor principal.

\section{DESARROLLO}

Haciendo un análisis de las publicaciones de referencia sobre la didáctica del FLE de los últimos 15 años y de los principales editoriales de mayor distribución en Francia y en América Latina, se pudo constatar que le dedican poco espacio a la enseñanza de la fonética y/o de la pronunciación o ni siquiera se refieren a ella (Vigner, 2001; Porcher, 2005; Bertocchini y Costanzo, 2008). En cuanto a Courtillon (2003: 57), se refiere muy brevemente a la existencia de ejercicios que ayudan a oír las diferencias entre los fonemas cuya percepción influencia la comprensión oral, pero ya advierte que son paliativos y que no pueden sustituir la práctica constante de escuchar.

Cuq y Gruca, $(2002 ; 2005 ; 2008)$ en su manual sobre la didáctica del francés como lengua extranjera o segunda y de reconocida referencia para la formación de los profesores de FLE, se refieren a la fonética en su capítulo sobre metodología de la expresión oral y afirman que la corrección de la pronunciación ha sido un preocupación constante en la enseñanza de los idiomas, pero, como otros autores, entre ellos Guimbretière, (2002), Germain, (2000) y Billières, (2008) advierten a los futuros docentes que la enseñanza de la fonética suele resultar aburrida para los alumnos e ingrata para los profesores, aunque no se puede obviar ya que condiciona en primerísimo lugar la comprensión y la expresión orales. 
Para facilitar la tarea de los profesores, observan que todos los métodos actuales de francés dedican un espacio a la pronunciación y que los materiales complementarios se esfuerzan en proponer ejercicios de corrección fonética prácticos y amenos, focalizados sobre la forma e inspirados de las diferentes teorías de la fonética correctiva. Cuq y Gruca, $(2002$; 2005) explican a los futuros docentes que existen cuatro grandes estrategias tradicionales de corrección que, de un modo u otro, se van perpetuando en los manuales de francés desde hace varias décadas: los métodos de articulación, de oposiciones fonológicas, el verbo-tonal y finalmente de enfoque prosódico.

El método articulatorio se base en el conocimiento profundizado del aparato de fonación y parte del principio que hay que conocer las características de un sonido y su punto exacto de articulación para poder pronunciarlo correctamente. Para aplicar esta estrategia que consiste en explicar los problemas y los mecanismos articulatorios, se utilizan esquemas de la cavidad de la boca, la nariz y la faringe para describir cómo redondear más o menos los labios, dónde colocar la lengua, abrir o cerrar la mandíbula, activar o no las cuerdas vocales. Esta información ha de permitir al alumno adecuar su articulación para producir correctamente un sonido determinado. Se trata de una apropiación teórica del sistema fonológico de la lengua estudiada que orienta su aprendizaje hacia un trabajo "físico” de la voz y una práctica consciente de la producción de los sonidos.

Por su parte, Billières, (2008) tampoco está conforme con los principios del método articulatorio según los cuales un aprendiz de lengua sólo puede repetir los sonidos si sabe cómo articular pero reconoce que todavía está muy utilizado para enseñar pronunciación. Advierte que los profesores de FLE han de conocer sus carencias, entre las que se pueden citar las siguientes: focalizado en la articulación, deja de lado la modalidad auditiva de la lengua que es preponderante; propone descripciones fijadas de los sonidos, cuando la palabra es ante todo movimiento, y los órganos articulatorios se mueven en sintonía con el cuerpo entero; tiende a crear en el alumno un reflejo articulatorio único cuando los sonidos del habla se influencian entre sí dentro de las sílabas y se escapan de las descripciones canónicas que propone el método articulatorio. Este mismo autor confiere gran importancia a la prosodia que es crucial para el habla y los ejercicios generalmente basados en listas de palabras son descontextualizados, fundamentalmente la utilización del método verbotonal (MVT) de corrección fonética basado en el principio de que una mejor percepción entrena una mejor producción.

Lo que es cierto es que con el empleo adecuado del MVT, el docente podrá recurrir a varios procedimientos para tratar un mismo y solo error, con la utilización de los gestos co-verbales y dando prioridad al ritmo y a la entonación.

En cuanto a Alliaume, (2005), la concientización de los puntos de articulación ha demostrado ser poco eficiente ya que la acción de los órganos vocales cuando se habla es tan inconsciente como los procesos de respiratorios. 
El método de las oposiciones fonológicas consiste en la oposición de fonemas y acarrea un aprendizaje mediante pares mínimos y ejercicios de discriminación esencialmente de tipo binario. Abry, autora de varias manuales sobre corrección fonética, explica en su último manual sobre fonética (Abry y Veldeman-Abry, 2007) que este método relaciona la fonología por una parte con la morfología, y por otra parte, con el vocabulario, insistiendo en los fonemas utilizados como marcadores morfológicos y de léxico. Los alumnos han de adquirirlos para comprender y luego producir una estructura o un lexema.

Para Cuq y Gruca, (2002; 2005), el enfoque prosódico o enfoque rítmico derivado de la teoría verbo-tonal constituye un cuarto método de corrección fonética que se aposentó durante la segunda generación de la metodología SGAV. Prioriza el aprendizaje del ritmo y de los esquemas melódicos para la percepción y la producción. Este planteamiento defiende que la adquisición de las estructuras prosódicas facilita la de los elementos fonéticos y contribuye a dar un sentido general de la interpretación. Abre una nueva vía ya que asocia la entonación con actitudes y con expresiones faciales o gestuales, y conduce a nuevas orientaciones didácticas inspiradas de las prácticas teatrales que fusionan los planos fónicos, prosódicos y gestuales, y sobre todo el sistema fono-gestual. El gesto, a semejanza de la entonación, entrega un mensaje verbal. Gesto y palabra van íntimamente ligados, favorece la interpretación pero también pueden ayudar a la expresión.

La "gestuelle du corps" está identificada como una estrategia de corrección de la pronunciación por Abry y Vedelman (2007) y explican:

"Lorsque l'apprenant a du mal à assimiler ou à produire des sons nouveaux pour lui, ceux qui n’appartiennent pas à son système phonologique (en particulier les voyelles nasales), on peut faire intervenir la gestuelle, impliquer tout le corps dans l'effort de prononciation ${ }^{1}$ ".

Llisterri, (2003) sitúa la enseñanza de la fonética fuera del ámbito común de la enseñanza de una lengua extranjera, por ejemplo, en un nivel muy avanzado o en unos estudios de filología. En cambio, incluye la enseñanza de la pronunciación dentro de las actividades de aula.

Para Bartoli, (2005) es evidente que enseñar fonética no es lo mismo que enseñar pronunciación y que los alumnos no necesitan estudiar los sonidos de una lengua extranjera sino saber pronunciarla.

Cantero, (2003) aclara que la fonética es el estudio de la forma material del habla, es decir de los sonidos que intervienen en la comunicación humana. La fonética acústica estudia cómo son los sonidos, la fonética articulatoria consiste en el estudio de cómo se pro-

\footnotetext{
${ }^{1}$ Cuando el alumno tiene dificultad para asimilar o producir sonidos que son nuevos para él/ella, aquellos que no pertenecen a su sistema fonológico (especialmente las vocales nasales), se pueden utilizar gestos, todo el cuerpo puede estar involucrado en el esfuerzo de pronunciación.
} 
ducen y la fonética perceptiva, se centra en cómo se perciben los sonidos durante los procesos comunicativos. En cambio, la pronunciación es la producción y la percepción del habla. Según Cantero, la enseñanza de la pronunciación es pues la aplicación inmediata de la fonética, pero advierte que hemos de dejar de confundirla con la corrección fonética.

Cuq y Gruca, (2005), afirman que pronunciar es ser capaz a la vez de entender y producir los sonidos, el ritmo y la entonación de la lengua y consideran esencial ligar íntimamente en la enseñanza y el aprendizaje del sistema fonológico y prosódico, la audición o percepción con la articulación. Existe un estrecho vínculo entre ambas: un fonema o un elemento prosódico no puede ser producido si no está percibido, pero al mismo tiempo, el gesto articulatorio es necesario para percibir, el hecho de producir o de articular (o de intentar articular) un sonido, ayuda también a su percepción. Cuq y Gruca, (2005) concluye que la pronunciación ha de tener un papel importante en el tratamiento didáctico de la oralidad considerada como un acto verbal que se realiza dentro de una relación interactiva entre al menos dos personas.

Por su parte, Iruela, (2007) argumenta que la pronunciación se encuentra en todas las actividades comunicativas de la lengua: expresión oral, comprensión auditiva, interacción oral y lengua escrita.

Según el Marco Común Europeo de Referencia para las Lenguas (ahora MCER), para permitir los procesos comunicativos de la expresión oral, el alumno ha de saber "articular el enunciado (destrezas fonéticas)" (MCER, 2002:88). En efecto, la pronunciación es el soporte de transmisión de la información oral, de forma que puede facilitar o dificultar al interlocutor el reconocimiento de las palabras. Por tanto, para Iruela (2007) "la importancia comunicativa de la pronunciación reside en que otorga la inteligibilidad al texto oral del que forma parte." Seguidamente, recuerda que para escuchar, el alumno necesita "percibir el enunciado (destrezas fonéticas auditivas)" (MCER, 2002:88), por lo que la pronunciación interviene decisivamente en la comprensión auditiva ya que es el soporte a través del cual se percibe la lengua oral.

Así pues, la didáctica de la pronunciación según Cantero (2005), es el enfoque metodológico que integra la pronunciación en los parámetros del enfoque comunicativo. Propone un tratamiento global de la lengua oral. Se centra en la expresión y la comprensión global en su conjunto, y no en la producción o la discriminación de sonido. Su principal objetivo ya no es la "pronunciación correcta" sino la adquisición de la competencia fónica de la lengua meta.

Es importante que los profesores de FLE estén familiarizados con los ejercicios y las técnicas de enseñanza de las habilidades fonéticas de recepción y de producción y con el material pedagógico que tienen a su disposición, para lo cual el autor propone una progresión didáctica que consta de siete fases o etapas estrechamente relacionadas con la definición anterior:

1) de sensibilización (auditiva y de representación visual de la articulación); 
2) de discriminación auditiva (de sonido, ritmo e entonación);

3) de integración corporal o articulación (imitando al profesor);

4) de asociación de la estructura fónica con su representación visual (códigos orales y escrito y perfiles melódicos);

5) de producción dirigida (calentamiento, repetición y transformación);

6) de producción espontánea o interpretación (breves presentaciones o debates).

Para el tránsito por las fases anteriormente referidas, se hace necesario el conocimiento, por parte de los docentes, de los componentes de la pronunciación que describe Lauret (2017:11). Para describir los componentes de la pronunciación, este autor describe dos niveles:

1. El nivel segmental se ocupa de los segmentos mínimos del habla:

- vocales (centro de la sílaba: una sola vocal por sílaba fonética);

- semiconsonantes o semivocales (llamadas así porque, aunque de naturaleza vocal, tienen un estado consonántico en la composición de la sílaba, preceden a la única vocal de la sílaba fonética en francés o a veces la siguen);

- consonantes y grupos de consonantes.

2. El nivel suprasegmental o prosódico, constituido por los parámetros siguientes:

- Ritmo: duración (regularidades rítmicas, pausas rítmicas, estiramientos, pausas);

- Acentuación: las sílabas marcadas por la duración y/o la altura y/o la intensidad;

- Entonación: melodía (ascendente, estable-alta o baja, descendente). Estos parámetros prosódicos tienen como soporte mínimo los grupos rítmicos compuestos de sílabas.

También se pueden abordar en este nivel, la cuestión de la naturaleza de la voz, los registros vocales y los timbres de la voz. Los aspectos que caracterizan cada nivel deberán ser tenidos en cuenta por el profesor durante la preparación de la clase y la relación armónica de todos sus componentes.

Los parámetros antes descritos, permiten considerar que el proceso de aprendizaje del FLE necesita de un análisis fonético, que a su vez, siente las bases para un entrenamiento fonético profundo, considerado este como un método que conduce a los estudiantes a realizar las acciones necesarias en el habla. Esto incluye:

- ejercicios con los órganos que intervienen en el proceso de fonación; 
- un entrenamiento auditivo (dictados frecuentes de palabras sin sentido construidas con sonidos de la lengua materna);

- aprender a utilizar los sonidos apropiados en palabras de las oraciones dadas, y

- aprender a producir secuencias de sonidos con el ritmo, la acentuación y la entonación correctos.

Lo anterior confirma lo oportuno del empleo de las técnicas para la corrección fonética, destacándose como fundamentales las siguientes: el método articulatorio, la repetición, la imitación y la oposición fonológica. Se debe priorizar también el uso del método verbotonal.

Como ejemplo de la aplicación de los presupuestos teóricos expuestos anteriormente pueden sugerirse tres actividades que fueron propuestas por los autores y aplicadas a tres grupos de estudiantes de la carrera Licenciatura en Educación, Lenguas Extranjeras, que estudian el francés como segunda lengua extranjera en la Universidad de Sancti Spíritus "José Martí Pérez", de Cuba. Los estudiantes tienen un nivel A1 según el Marco Común Europeo de referencia para las lenguas. Las actividades fueron aplicadas en sesiones de corrección fonética y en talleres de corrección fonética respectivamente que forman parte de la asignatura "Práctica Integral de la Lengua Francesa II".

\section{Actividad 1}

Tema: Discriminación del sonido [y]. Esta actividad permite trabajar los rasgos segmentales al discriminar el sonido.

Objetivo: Discriminar adecuadamente el sonido [y]

Medios: CD Audio y presentación en power point (PP).

Procedimientos: El profesor recordará los símbolos fonéticos del Alfabeto Fonético Internacional (API por sus siglas en francés). Los estudiantes los mencionan y ejemplifican. Luego el profesor presenta en PP el API para que los estudiantes se auto corrijan.

El profesor orienta a los estudiantes escuchar atentamente el documento audio y les hace las siguientes precisiones:

Escuche las frases. Cuando escuchen el sonido [y] en la primera sílaba, muestren la tarjeta con el número 1 , cuando escuchen el sonido en la segunda sílaba, muestren la tarjeta con el número 2 y cuando escuchen el sonido en la tercera sílaba, muestren la tarjeta con el número 3. Observen el ejemplo:

Ejemplo: Salut Anne! (3)

1. Oh, salut!

2. Tu vas bien?

3. Oui, c'est Luc. 
4. Luc, ça va?

5. À plus tard!

6. Oui, à plus!

Ejecución: Los estudiantes escuchan el documento audio y levantan la tarjeta según corresponda.

Control: El profesor pide a los estudiantes de autoevaluarse.

Otra actividad que se propone es la relacionada con la sílaba tónica.

$\underline{\text { Actividad } 2}$

Tema: Reconocimiento de los diferentes sonidos y oposiciones fonológicas.

Objetivo: Reconocer las oposiciones fonológicas a través de frases escuchadas.

Medios: CD audio.

Procedimiento: El profesor pregunta a los estudiantes cuáles son las oposiciones fonológicas que existen en francés y que no existen en nuestra lengua materna.

Los estudiantes mencionan ejemplos.

El profesor entrega el siguiente ejercicio: Marque la frase que usted escucha.

Los estudiantes escuchan el documento audio y marcan la frase que escucharon.

1. a) Elle est pure. b) Elle est pire.

2. a) Voici les faits b) Voici les fées.

3. a) Quel joli nez! b) Quel joli nœud!

4. a) Tu veux ce libre? Je te le prête b) Tu veux ces livres? Je te les prête.

5. a) Il y a deux amis. b) Il y a des amis.

6. a) Vous n'êtes pas sourds. b) Vous n'êtes pas sûrs.

7. a) Elle montre un dé. b) Elle montre un dais.

8. a) Elle a une bonne vie. b) Elle a une bonne vue.

Ejecución: Los estudiantes señalan la frase que escuchan.

Control: Se realiza la autocorrección por parte de los estudiantes y luego se indica que vayan a la pizarra a escribir la frase que escucharon en cada caso.

Por último, se propone la actividad número tres está relacionada con los rasgos prosódicos, en este caso con el fenómeno del enlace (liaison). 


\section{Actividad 3:}

Tema: El sonido [z] y el enlace verbal con los pronombres nous, vous, ils, elles.

Objetivo: Distinguir los sonidos [z]- [s] en los enlaces verbales de modo que contribuya al logro de la entonación de la frase.

Medios: CD audio.

Procedimiento: El profesor recuerda a los estudiantes el procedimiento para la transcripción fonética y su importancia para el trabajo con el diccionario.

¿Cuáles son los pronombres personales en francés? ¿Cómo se forma el enlace?

Se les pide que escuchen los verbos en el documento audio y que escuchen el sonido pronunciado cuando hay un enlace y cuando no lo hay.

Los estudiantes deberán realizar la transcripción fonética, según corresponda:

1. nous avons/nous savons

2. ils sont/ils ont

3. vous savez/vius avez

4. elles ont/elles sont

Ejecución: Los estudiantes, apoyados en el conocimiento del API podrán hacer la diferencia entre las oposiciones fonológicas $[\mathrm{z}]-[\mathrm{s}]$ y podrán realizar la transcripción fonética de los verbos que escucharán en el documento audio.

Control: Se evalúan individualmente a los estudiantes, por parte del profesor o de otro estudiante designado. Se enfatizan las conclusiones. Se señalan los errores, de este modo se rectifican las operaciones en las que demostraron menos o más dominio.

\section{CONCLUSIONES}

El profesor de LE debe reconocer la importancia de la lengua oral como medio de expresión y comunicación y de la pronunciación como su soporte; esa es la premisa fundamental para que se preocupe por incluirla (a la segunda) en el proceso de enseñanza-aprendizaje. El hecho de que el proceso de enseñanza-aprendizaje de la LE se realice en su medio lingüístico, no exime al profesor de la responsabilidad de desarrollar la competencia fónica del estudiante. Para esto, debe concebir la pronunciación como una habilidad que ha de enseñarse y desarrollarse en ese proceso.

Los adelantos científicos y técnicos, la aparición del Internet, la web participativa e interactiva y de los soportes digitales en general, demandan cada vez más la necesidad de transformar la enseñanza de la de las LE en la actualidad, lo que constituye una vía idónea para hacer de la clase un espacio propicio en cuanto a la apropiación, por parte de los 
alumnos, de nuevos métodos y herramientas en aras de mejorar su aprendizaje. Las T.I.C. constituyen recursos que el profesor de LE no debe obviar y ponerlos en función del diagnóstico de sus estudiantes y emplearlas como soporte complementario en las clases.

\section{REFERENCIAS}

Abry, D. \& Veldeman-Abry, J. (2007) : La phonétique. Audition, prononciation, correction. Paris: CLE International.

ADELL, J. (2006): «Riesgos y posibilidades de las TIC’s en educación.» Conferencia XXI Semana Monográfica sobre educación en la Fundación Santillana de Madrid, 20-24 de noviembre 2006. Disponible en Web: [Enlace]

Alliaume, J. (2005): Parole(s) de cerveau. Neuro-pédagogie de la parole. Québec : Fondation littéraire Fleur de Lys.

BARTOLI, M. (2005): « La pronunciación en la clase de lenguas extranjeras »PHONICA : 1-27.Disponible en Web: [Enlace]

Bertocchini, P. \& COSTANZO, E. (2008): Manuel de formation pratique pour le professeur de FLE Paris : CLE International Vigner, G. (2001): Enseigner le français comme langues seconde. Paris: Clé-International, p.67

Billieres, M. (2008): «Où en est la méthodologie du français langue étrangère.» Eventos $n^{\circ}$ IV, Publicación de la Universidad Central de Venezuela, Caracas: 55-66.

BRouTE, A. (2008): « La modernisation de l'enseignement-apprentissage des compétences de production orale en FLE ne peut plsu attendre » Synergie Espagne no 1 : 213-221. Disponible en Web: [Enlace].

CANTERO, F. J. (2003): Oír para leer: la formación del mediador fónico en la lectura. Madrid: Ministerio de Educación, Cultura y Deporte - Colección Aulas de Verano

CANTERO, F. J. (2005): « Comunicació i veu», en ARTICLES de Didàctica de la Llengua i la Literatura, num. 32. (Monografia: Veu i locució).

CONSEIL DE L'Europe (2002). Cadre Européen Commun de Référence pour les Langues. Apprendre, enseigner, évaluer. Paris, Didier.

COURTILlON, J. (2003): Elaborer un cours de FLE. Paris: Hachette-Français langue étrangère.

CUQ, J.P. \& GRUCA, I. (2002, 2005, 2008): Cours de didactique du français langue étrangère et seconde. Grenoble: PUG.

Demaiziere, F. \& NARCY-COMBES, J.P. (2007): « Méthodologie de la recherche didactique : nativisation, tâches et TIC». Alsic, Vol. 8. no 1 : 45-64. Disponible en Web: [Enlace] 
Germain, A. \& Martin, P. (2000): « Présentation d'un logiciel de visualisation pour l'apprentissage de l'oral en langue seconde. » ALSIC, Vol. 3, nº $1: 61-76$.

Guichon, N \& Penso, A. (2002): « Vers une appropriation des outils multimédias. » Les Cahiers de l'APLIUT Vol. XXI - N³ Disponible en Web: [Enlace]

Guimbretiere, E. (2000). L'enseignement de la phonétique : état des lieux entre tradition et modernité. Mélanges (Crapel) n²5 : 153-168. Récupéré sur: [Enlace]

Hirschsprung, N. (2005): Apprendre et enseigner avec le multimédia. Paris : CLE International.

Iruela Guerrero, A. (2007): « ¿Qué es la pronunciación? ». Revista Electrónica de Didáctica ELE, No. 9. Disponible en Web: [Enlace]

LAURET B. (2017). Enseigner la prononciation du français : questions et outils, Paris, Hachette FLE.

LLISTERRI, J. (2007): « La enseñanza de la pronunciación asistida por ordenador ». En Actas del XXIV Congreso Internacional de AESLA. Aprendizaje de lenguas, uso del lenguaje y modelación cognitiva: perspectivas aplicadas entre disciplinas. Madrid: Universidad Nacional de Educación a Distancia, 91-120. Disponible en Web: [Enlace]

MANGENOT, F. (2003). «Tâches et coopération dans deux dispositifs universitaires de formation à distance ». Alsic Vol. 6, №1, Juin 2003, pp. 109 - 125, http://alsic.org

MESSAOUDI, F. (2010): « Introduire l'innovation comme système d'amélioration de l'enseignement du FLE». En Guillén, C. (Coord.) Francés: investigación, innovación y buenas prácticas. Vol. III: 7796. Barcelona: Graó.

PorCHER, L. (2005): L’enseignement des langues étrangères. Paris: Hachette -Éducation.

VERA, C. (2010): «Les matériels didactiques pour la classe de français langue étrangère.» En Guillén, C. (Coord.) Didáctica del francés. Vol. II.. Barcelona: Graó, pp. 93-106

VignER, G. (2001). Enseigner le français comme langue seconde. Paris: CLE International. 